\title{
Underwater Self Deployment of Wireless Sensors for Maximum Coverage and Connectivity using K-Means Clustering
}

\author{
Gurjaspreet Kaur ${ }^{1}$, Surinder Singh ${ }^{2}$, Ramanpreet $\operatorname{Kaur}^{3} \&$ Gaurav Garg ${ }^{4}$ \\ ${ }^{1,4}$ Department of Electronics and Communication, CEC Landran, Punjab, India \\ ${ }^{2,3}$ I.K.G Punjab Technical University, Punjab, India \\ *Corresponding author E-mail: nirwangurjas@gmail.com
}

\begin{abstract}
By virtue of abundant research being executed in terrestrial wireless sensor networks, we get enlightened about the different advantages of using wireless sensors and implemented these wireless sensors in underwater to probe the area below the sea for its various applications. The most crucial task in underwater 3D space is the node deployment such that sensors can cover the maximum area while simultaneously maintaining the connectivity with the base station. Due to the advantages of self deployment where no pre calculations and no human interventions are needed, we proposed a distributed move restricted self deployment underwater wireless sensor network. The main emphasis is on maximizing the coverage area by the sensor nodes and simultaneously maintaining the connectivity with the base station. Firstly, the maximum coverage has achieved by removing the interference between the sensor nodes which minimize the coverage overlap and thus more area can be covered which improves the network coverage. Then by using K-Means clustering, all the sensors nodes were divided into clusters, having one centroid for each cluster. Further all these centroids have been interconnected and then by finding the centroid which is nearest to the sink by Euclidean distance formula, we connect it to the sink.
\end{abstract}

Keywords: Connectivity; K-Means Clustering; Maximum Area Coverage; Movement Restricted Deployment; Self Deployment; Underwater Communication.

\section{Introduction}

There had been a vast amount of analysis done in wireless sensor networks, from where we come to know the various advantages of using wireless sensors. Wireless data communication through the ocean is one of the empowering advances for the improvement of future ocean perception frameworks and sensor systems. Water volumes covered $70 \%$ of the earth's area, out of which only $10 \%$ has been investigated and the rest is still unexplored. An extensible underwater sensor network (UWSN) gives a promising solution for finding the aqueous environment proficiently and examine such area for distinctive applications. Applications of UWSN compass oil industry to aquaculture, pollution control, prediction of natural disturbances, climate recording, study of marine life, survey and search missions. [1-3]

Nowadays mainly acoustic technology has been used for the underwater communication systems whereas for the short-range links (typically 1-10 m), the other communication techniques like optical and radio frequency, or even electrostatic communication have been proposed, where their high bandwidth in $\mathrm{MHz}$ or even more can be exploited. But high power or large antennas are required because these signals get attenuated rapidly within tens of meters (optical) or few meters (radio).[4]

UWSN contains huge number of sensor nodes deployed in the ocean which communicate with each other by emitting acoustic waves. These sensor nodes are in charge of gathering the detected data and after that hand-off it to surface station floating on the surface of the ocean. The networked sensors coordinate over large scale of physical space to perform disseminated sensing of envi- ronmental phenomena and enable reliable monitoring and control in different applications.

UWSNs for the most part include parts of underwater communication technology, reliable data transmission, node deployment, network protocol design, localization for underwater and tracking of target, power management, synchronization of time, storage management and underwater security. Deployment is a standout amongst the basic concern in UWSNs in light of the fact that it doesn't just promote underwater monitoring quality, additionally reduces network development cost. Moreover, it supports the network topology control, boundary detection and routing. A proper node deployment plan be able to likewise a great establishment for resulting network communication protocol designs and operations. Thus, building strategies to deploy nodes is a basic issue that ought to be earnestly tended to. Node deployment in UWSNs is unique in relation to those of node deployment of wireless sensor in terrestrial systems. For instance, a node is by and large conveyed in $3 \mathrm{D}$ space in the former and thus faces new challenges in terms of mobility, connectivity and coverage. Conferring to the movement ability of nodes, underwater node deployment algorithms can be divided into static and self-deployments $[5,6]$. In static deployment, the position of the nodes is needed to be precalculated and is deployed manually on predetermined locations [7]. But in numerous UWSN cases, such as underwater resource exploration, tactical surveillance and where the amount of the monitored area is large, then this manual deployment will require significant extra manpower and material assets. Thus static deployment is not suitable for such cases. On the other hand in self deployment, there is no need of pre-calculations and the nodes can shift and repositioned dynamically according to the needed topology to reach the target without any human intervention. Self de- 
ployment further on the basis of movement capabilities of nodes, can be classified into move restricted and free to move deployment algorithms. In move restricted deployment, nodes can only move in specific direction, usually vertical and its corresponding algorithms are called depth adjustment algorithms. While in free to move deployment, it is assumed that the can reach any position by moving freely in the water. Among both these self deployment techniques, move restricted deployment is more realistic than free to move deployment because in UWSN, free to move deployment required an autonomous underwater vehicle for the adjustment of network topology which results to high energy and resource cost contrarily in the move restricted self deployment underwater sensor nodes have vertical mobility in which the nodes can move up or down by the technique of drawing of or filling in the water respectively which is a simple and accessible process. In this paper, we proposed a distributed move restricted self deployment underwater wireless sensor network which emphasis on maximizing coverage by the sensor nodes while simultaneously maintaining the connectivity with the base station by using K-Means. On one hand, various correlated studies have been conducted on the technique for move-restricted node self-deployment. In 2009 akkaya et al. [7] by using the distributed node deployment scheme, increased the network coverage on iterative basis. Nodes were initially deployed at the bottom of water which then relocates themselves at different depths by moving in vertical direction in 3D space of water to reduce the sensing overlap among the neighboring nodes. This procedure of adjusting the node depths continue until no further improvement in the coverage can be done. Also it provides the connectivity of the sensor network with the base station.

In 2014 hua et al.[8] proposed improved self organize map algorithm for the seabed nodes arrangement .In this, victory nodes were selected based upon their distance from the events and if this distance is long then these nodes will be abandoned. Self organize map algorithm thus provides the probability selection mechanism to select victory nodes in Gibbs sampling. The nodes far away from the events were then moved to the direction of events, after the nodes near the events become the victory nodes.

In 2014 xia et al.[9] proposed the particle swarm inspired underwater sensor self deployment in which the flying behavior of particles was simulated and the sensors were drive to cover all the events and avoid overcrowding simultaneously.

In 2015, jiang et al.[10] proposed a node deployment algorithm based on connected tree which focus on maximum coverage by reducing the coverage overlaps between the neighboring nodes by constantly adjusting the depth of nodes. In connected tree depth adjustment algorithm (CTDA), sink node is considered as the root node to initiate the structure of the connected tree. Finally by organizing the network in form of forest, the connectivity can be maintained. For optimize coverage, the overlaps between the parent and child node were reduced within each sub tree.

In 2016 jiang et al.[11] proposed an uneven clustering with radius adjustment. In this algorithm the nodes initially begin the uneven clustering based on their distance from the surface of water. Then the clusters heads choose their next hop to make a connected pathway to the sink node using hybrid radius path selection method. After that each cluster head node adjust the depth of the in cluster nodes and then find the next cluster to be adjusted.

\section{Network Model and Assumptions}

Initially it was assumed that $\mathrm{n}$ sensor nodes were randomly spread on the water surface with each node has communication, perception and mobility capabilities (vertical direction). By moving these nodes, their position can be adjusted underwater to structure a 3D coverage network. Another assumption has been made that these sensor nodes can sense a spherical space with sensing radius $r$, which is constant for every node and the centre of the sphere is the location of that particular node. Any point $\mathrm{P}$ outside the radius $\mathrm{R}$ of the sphere cannot be covered by that node.
It was assumed that an extensive number of underwater sensors are scattered in a region to be monitored with a surface station. The location of sensors and surface station was assumed to be known by ranging or range free mechanism [7]. Since the underwater sensors which used here were self reconfigurable, it was assumed that they have the ability to change their positions by their own through mobility. However the mobility of these sensors is limited as per the present innovation, these sensors can only move in the vertical direction (i.e. $\mathrm{z}$ axis), thus can only adjust their depths whereas the horizontal movement (i.e. $\mathrm{x}$ and $\mathrm{y}$ axis) is not possible except with the impacts of streams and waves. With the current technology, the depth of the underwater sensors can be adjusted in different ways. For example, in [4], a Mica2 mote was placed in a cylinder having a piston for forcing water out and drowning in the water. The sensor starts sinking when the water moves in and start rising in when the water moves out. Thus the sensor can adjust its depth in this way. Similarly, an air-bladderlike gadget is prescribed to be utilized as a part of underwater sensors for such reason in [11]. Another method used for the adjustment of depth of the sensors is to use tethers from anchors which are placed at the ocean's bottom. There is a buoy at every sensor, which can be inflated by an electronically controlled pump placed on the sensor. The length of the wire can then be changed in accordance with control the depth of the sensor.

\subsection{Algorithm for removal of interference for maximum coverage}

Fig. 1 shows the depth adjustment of the nodes unless there is no interference among them.

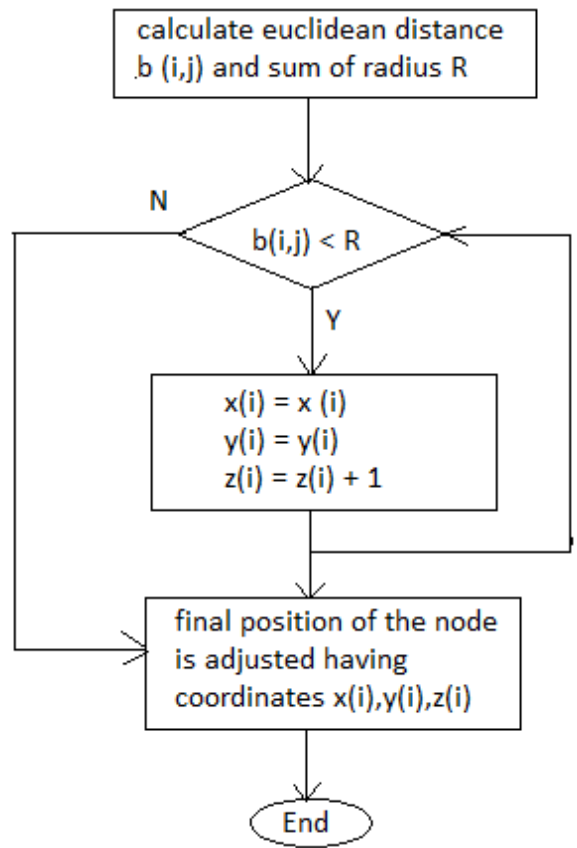

Fig. 1 Algorithm for the Removal of Interference between the Nodes

Initially the $\mathrm{n}$ numbers of sensors are deployed arbitrarily in the monitoring area of the ocean. The sensor nodes are considered to be appropriately deployed if they cover the maximum area and simultaneously maintain the connectivity with the sink node. If these two goals are achieved then only the sensor network is considered to be deployed accurately. Thus our first step is the maximum coverage area, which is achieved by removing the interference between the sensor nodes. By removing the interference between the sensor nodes, it minimize the coverage overlap and thus more area is covered which will improve the network coverage.

The algorithm we used for removing the interference is firstly finding the Euclidean distance between the sensor nodes. The Euclidean distance between every node is calculated by the formula following Eq. (1) 
$\mathrm{b}(\mathrm{i}, \mathrm{j})=\sqrt{ }(z(i)-z(j))^{2}+(y(i)-y(j))^{2}+(x(i)-x(j))^{2}$

where $b(i, j)$ is Euclidean distance; $x, y$ and $z$ are the coordinates of the location of the nodes in 3D space.

Now we compare this Euclidean distance with the sum of the constant radius $r$ of that two nodes. Where Sum of radius $R=r+r$

If the Euclidean distance is less than the sum of the radius, then there will be overlapping between the nodes and therefore the $\mathrm{z}$ coordinate of one of the nodes is thus increased by one. This step is repeated until the distance become equal or greater than the sum. When that position is achieved where the Euclidean distance is greater or equal to the sum of the radius that will be considered as the final coordinates of the node's location. As the sensor nodes we used are move restricted, they can move only in vertical direction therefore only $\mathrm{z}$ coordinates can be changed. Thus the depth of the nodes is adjusted by moving them in vertical direction until there is no overlapping. . Previously centralized method is used in which the depth of each node is calculated by the sink node. But the centralized method is difficult to be achieved by the distribution of the nodes. Thus we used a distributed method in which each sensor node estimates its location individually. Figure 2 shows the initial deployment of the nodes which have interference between them that leads to overlapping of the coverage area of the nodes .while figure 3 shows the removal of the interference after performing the algorithm.

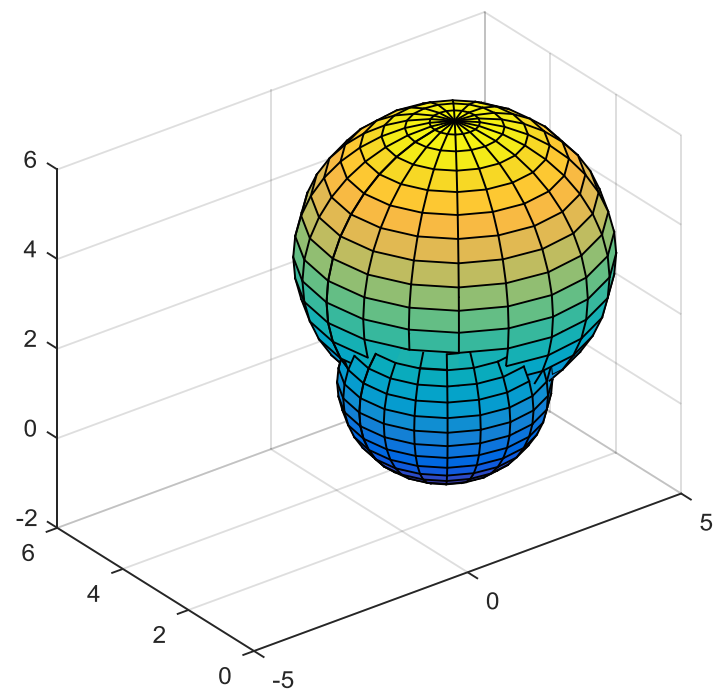

Fig. 2. Random deployment of nodes

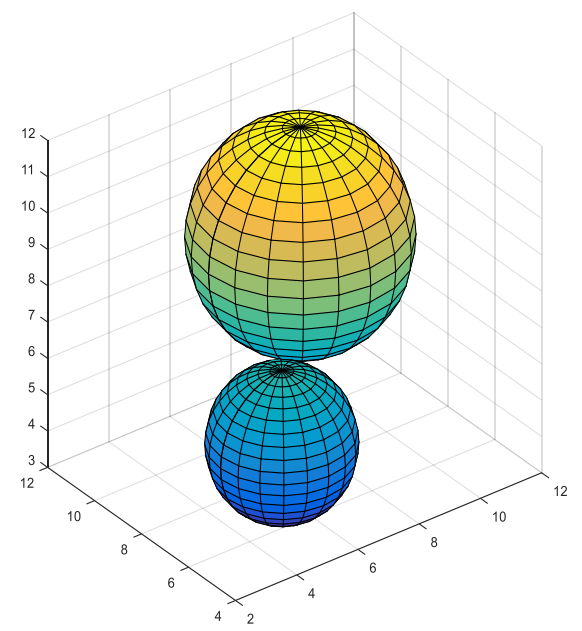

Fig. 3. Nodes after Removal of Interference

\subsection{Clustering}

After the removal of interference, the next task is the grouping of nodes so that each node is linked to the sink node. The clustering is done using $\mathrm{K}$-means in which all the observations partition themselves into $\mathrm{K}$ clusters and each observation belongs to the cluster with the nearest mean. The clusters thus formed, have very high intra cluster and very low inter cluster similarity. The number of clusters is defined by the $\mathrm{K}$ which is the positive integer number. The principle thought of K-Means is to define $\mathrm{K}$ centroids, one for every cluster. The initial step is to associate every point of the given data set to the closest centroid, in terms of Euclidean distance to the cluster mean. At the state when no point is pending, the initial step is finished and an early grouping is completed. Now we have to re-compute K new centroids as barycenters of the clusters formed in the previous step. After we have these k new centroids, another coupling must be done between the same data set points and the closest new centroid. It generated a loop, as a result of which $\mathrm{K}$ centroids changes their location step by step until no more changes are done. In spite of the fact that it can be demonstrated that the system will dependably end, the calculation is likewise altogether delicate to the initial arbitrarily selected cluster centre's. K-means is a straightforward calculation that has been adjusted to numerous issue domains.

We had deployed 50 sensor nodes in the area of $200 \mathrm{~m}$ and divide them into 12 clusters using $K$-means i.e. $K=12$ and $n=50$ with each sensor nodes having a constant sensing radius $\mathrm{r}$ of $10 \mathrm{~m}$. This simulation is done in matlab software. The 12 clusters thus formed, have one centroid for every clusters. All the nodes in a cluster are directly attached to its centroid and all the centroids are further connected to each other. Then by using the Euclidean distance formula we attached the nearest centroid to the sink. Thus information at every region get sensed by the node of the corresponding region and then delivered it to its centroid. The centroid further passed it to the base station for processing.

Fig. 4 represents the clustering of nodes in which the nodes numbered from 1 to 50 were connected to their corresponding centroids shown using red circles. The base station BS is connected with the nearest centroid.

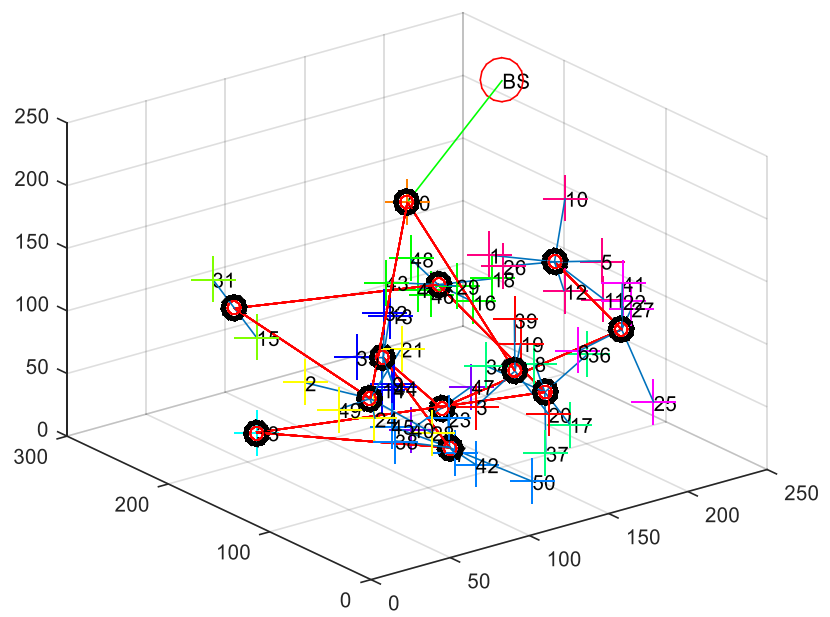

Fig. 4. Clustering of Sensor Nodes

\section{Simulation and Performance Analysis}

In this study we analyze, simulate and compare by the parameters of coverage and connectivity. The node deployment process in UWSN and the clustering is simulated using Matlab.

\subsection{Coverage rate}

The coverage rate of the network is defined as the coverage scale of an underwater sensor network of the monitoring area or the target and is also an essential standard to assess a node deployment algorithm. The coverage rate is calculated using the formula following Eq. (2)

$\mathrm{C}=\frac{V \operatorname{cov}}{V t t l}$ 
Where Vcov is the volume covered by the sensor nodes and Vttl is the total volume of the monitoring area in 3D space

In figure 5 the graph shows the comparison between the $\mathrm{k}$ means and the different self deployment techniques of SDDA, CTDA,CDA on the bases of the coverage rate by considering varying number of nodes. The results in the graph depicts that by increasing the number of nodes from 10 to 20 , the coverage rate of each technique increases simultaneously .Here the radius of every node is assumed to be $10 \mathrm{~m}$.

coverage comparison with varying number of nodes

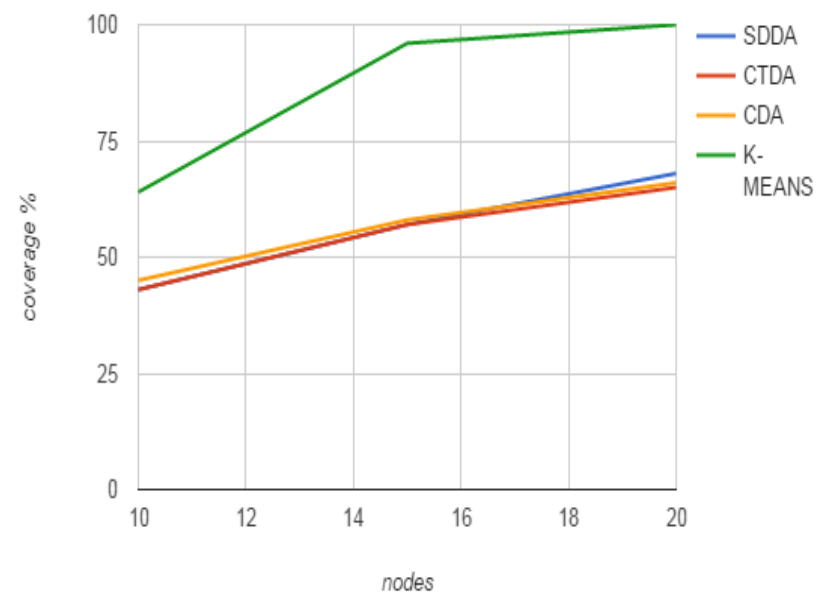

Fig. 5. Graph of coverage comparison with varying number of nodes of different techniques

\subsection{Connectivity rate}

It is the essential parameter which depicts the quality of service of the sensor network and is defined as the ratio of the number of nodes connected to the sink node to the total number of nodes, given by Eq. (3)

Con $=\frac{N \sin k}{N t t l}$

Where Nsink is the number of nodes connected to the sink and Nttl is the total number of nodes.

As we have used K-means clustering in which all the nodes are grouped into 12 clusters each having their own centroid and all the nodes in each cluster are directly connected to its centroid. Moreover all centroids are interconnected and the nearest is connected with the sink. Thus all the nodes are connected to the sink and gives $100 \%$ connectivity except the case if any of the centroid stop working. In that case all the nodes in that cluster will get disconnected from the sink.

\section{Conclusion and Future Scope}

Underwater sensor network deployment is considered the important research topic as it is very crucial in the terms of security in the military and the surveillance. With the help of UWSN the enemy submarines and other suspicious vehicles can be detected and attacks through the sea can be controlled. Moreover the research area of UWSN is very wide. It also includes industry, study of marine life, detecting the disasters, study of endangered species like coral reefs, etc. The efficiency of the UWSN depends on the deployment of sensors nodes. More efficiently the nodes are deployed, more will be the network reliability. The deployment we used is the move restricted self-deployment. The approach used here is distributed in which each node adjusts its own depth and is not dependent on the centralized hub, thus it is more advantageous and efficient than other methods. The deployment of sensors and their clustering are obtained using MATLAB software. The comparison between the different techniques for self-deployment is made on the parameters of connectivity and coverage rate which shows $\mathrm{k}$ means is more efficient than other methods.

\section{References}

[1] Climent, S, Sanchez, A, Capella, J.V, Meratnia, N., \& Serrana, J.J. (2014), Underwater acoustic wireless sensor networks: Advances and future trends in physical, MAC and routing layers. Sensors 14, 795-833.

[2] Guo, Z.W, Luo, H. J., Hong, F., Yang, M., \& Ni, M. X. (2010), Current progress and research issues in underwater sensor networks. J. Comput. Res. Dev. 47, 377-389.

[3] Ian. F. (2014), Wireless sensor networks in challenged environments such as underwater and underground. In Proceedings of the 17th ACM International Conference on Modeling, Analysis and Simulation of Wireless and Mobile Systems, New York, NY, USA, pp. 1-2.

[4] Heidemann, J, Stojanovic, M., \& Zorzi., M. (2012), Underwater sensor networks: Applications, advances and challenges. Phil. Soc. R. Trans. A 370,158-175.

[5] Liu, J., Han., X., Al-Bzoor, M., Zuba., M., Cui, J.H., Ammar R. A., \& Rajasekaran, S. (2012) PADP: Prediction assisted dynamic surface gateway placement for mobile underwater networks. In Proceedings of IEEE Symposium on Computers and Communications (ISCC), Cappadocia, Turkey, pp. 139-144.

[6] Cui, J.H., Kong, J., Gerla, M., \& Zhou, S. (2006), The challenges of building mobile underwater wireless networks for aquatic applications. IEEE Netw. 20, 12-18.

[7] Akkaya, K., \& Newell, A. (2009), Self-deployment of sensors for maximized coverage in underwater acoustic sensor networks. Comput. Commun. 32, 1233-1244.

[8] Hua, C. B., Wei, Z., \& Nan, C. Z. (2014). Underwater Acoustic Sensor Networks Deployment Using Improved Self-Organize Map Algorithm. Cybernetics and Information Technologies 14, 63-77.

[9] Du, H., Xia, N., \& Zheng, R. (2014). Particle swarm inspired underwater sensor self-deployment. Sensors 14, 15262-15281.

[10] Jiang, P., Wang, X., \& Jiang, L. (2015). Node deployment algorithm based on connected tree for underwater sensor networks. Sensors 15, 16763-16785.

[11] Jiang, P., Xu, Y., \& Wu, F. (2016). Node self-deployment algorithm based on an uneven cluster with radius adjusting for underwater sensor networks. Sensors 16, 98. 